\title{
Selection criteria of optimal characteristic material and technologies for precision processing of basic working surface of human hip-joint implant
}

\author{
Raul Turmanidze ${ }^{1, *}$, Predrag Dašić ${ }^{2}$, and Giorgi Popkhadze ${ }^{1}$ \\ ${ }^{1}$ Georgian Technical University, Department of Production Technologies of Mechanical Engineering,Kostava St. 77, Tbilisi \\ 0175, Georgia \\ ${ }^{2}$ Faculty for Strategic and Operational Management (FSOM), Str. Jurija Gagarina 149, 11070 Novi Beograd,
}

\begin{abstract}
In view of the fact that the endo-prosthesis heads of human hip-joint are operated in extreme conditions, in respect of load, the selection of corresponding material and also increase of precision and quality of machining of spherical surfaces is rather topical task. In the submitted work are reviewed the problems connected with definition of the influence degree of orientation of the sapphire crystal on its workability during diamond grinding with a butt of the ring and elaboration of the perspective, original scheme of formation of the incomplete spherical surface, particularly, of the sapphire head of endo-prosthesis of the human hip-joint.
\end{abstract}

\section{INTRODUCTION}

Endo-prosthetics is effective and often the only method of the function restoration of the human joint. It is established that every year in the world are made about a million operations of exchange of the human hip-joint.

The endo-prosthesis heads of the human hip-joint from the point of view of the character and value of load are operated in extreme conditions. The contemporary joint endo-prostheses consist of acetabular (cup) and hip component (leg) and the head from metals or ceramic materials on their base. At present, there is an acute problem of creation of wear resistant inert materials for Implant- ology.

Therefore, in each specific case the selection of the necessary material with corresponding physicalmechanical characteristics and also increasing of precision and quality of the most significant part of endoprosthesis - spherical surfaces is rather actual task the acuteness of which intensively grows in recent years. The number of used endo-prostheses is some tens of millions of pieces a year and the statistics shows that unfortunately this number increases every year.

The production of implants from bio-ceramic materials is rather profitable direction. Up to now, a powerful industry of fabrication of implants, tools and accompanying materials has been created and the western market of this product is evaluated in 2,5-3 billion Dollars a year. The basic developing and producing countries of implants are USA, Japan, Germany, France, Great Britain, Russia, Italy, South Korea, countries of Asia region and other countries.
It is conditioned by the fact that if earlier the necessity of similar operations was caused by the age factor of the man or traumatology fractures in recent two decades abruptly increased the number of patients at young age of 30-40 years of both men and women without any injuries and fractures. In opinions of physicians, the principal reasons of it are non-active way of life of youth, composition of contemporary artificial food products and metabolic disorder. All the above-mentioned reasons determine the number of used endo-prostheses in some tens of millions of pieces a year.

The medical practice proves that the repeated prosthetics of the human hip joint is connected with big problems. In many cases, the implementation of such operations becomes practically impossible. Therefore, the durability of the endo-prosthesis of the human hip joint to the end of the patient's life especially at young age has especially significant meaning.

The contemporary elaborations of the endoprosthesis structure are directed to exchange of chirulen by ceramic material that leads to the change of the endoprosthesis structure, development of new ceramic materials with the improved physical and mechanical characteristics, development of processing of internal and external spherical surfaces, development of diamond tools providing the high quality of the machined surface, definition of the optimal wear resistance of the pair of materials for manufacture of the endo-prosthesis etc. For nowadays in the world practice these heads are manufactured from various alloys, composite materials and ceramics, which mainly are isotropic materials. Therefore, the data of the above-mentioned works do not give the necessary information on machining of

* Raul Turmanidze: inform@gtu.ge 
anisotropic materials, particularly artificial crystal of sapphire.

Together with indisputable positive qualities, the contemporary structures of endo-prostheses from metal, ceramics polymers have certain defects. Among them there is an insufficient biological inertness and redundant wear of components of the friction pair that lead to short duration of work of the artificial hip-joint. Besides that as the result of wear of the material in the friction pair of endo-prosthesis hinge toxic and oncological danger products of the dissociation in various organs and tissues accumulate that in $30-40 \%$ leads to hard complications and requires complex disabling interferences $[1,2]$.

The circle of materials that satisfy the criterion of biological compatibility is rather limited. Because of it and requirements of the resource of articles in condition of action of alternating loads, corrosion active environment titanium and its alloys as the material for fabrication of endo-prostheses have the advantage over Co-Cr-Mo alloys.

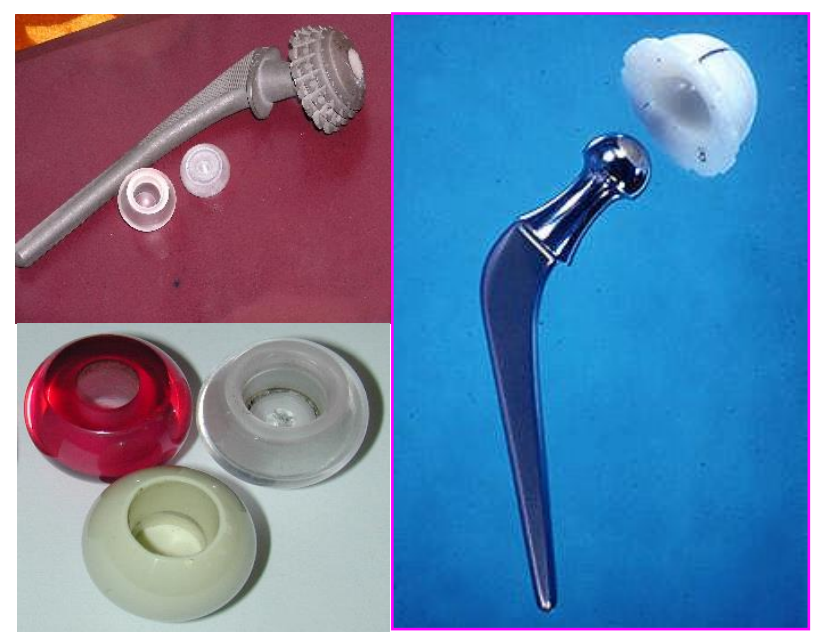

a

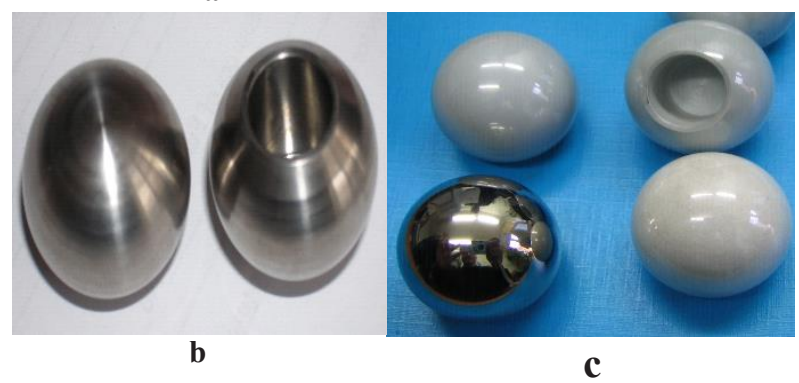

Fig. 1. Hip heads from sapphire a) zirconium ceramics

b) biologically pure titanium alloy c) stainless steel (SS)

\section{Grind ability of monocrystalline sapphire, experimental results and discussions}

The wear of heads from zirconium ceramics is 7-9 $\mathrm{nm}$ a year while titanium $105 \mathrm{~nm}$ a year. Coming out of this the fabrication of implants from bio-ceramic materials is a perspective direction of development of the science intensive technologies of creation of materials and their machining. Most biocompatible with the human organism, wear resistant and durable material for fabrication of the above-mentioned article is the artificial mono-crystal of sapphire.

For nowadays, the circle of materials for fabrication of pairs of bearing surfaces of endo-prostheses of joints with minimally possible number of products of wear has been determined. Such pairs of bearing surfaces with excellent characteristics of wear resistance are the friction pairs of ceramics-ceramics, metal-metal and polyethylene with a high degree of cross-section links in combination with ceramics or metal. Together with that, the basic unresolved problem of the last decade was the development of bearing surfaces that could endure much higher loads with young and movable patients. The surfaces that are being investigated at present in laboratory conditions due to their hopeful characteristics of wear are ceramic matrix ( $82 \%$ of aluminum oxide, $17 \%$ of zirconium dioxide, $0,3 \%$ chrome oxide), zirconium dioxide and ceramics in pair with cobalt-chrome alloy.

Together with that sapphire representing the monocrystal of aluminum oxide as a material for bearing surfaces possesses unique inertness including electrolyte passiveness, perhaps the best of the well-known materials, biocompatibility, corrosion resistance and hardness. Resistance of sapphire towards any acids and alkali is rather higher than that of metals and even poly-crystalline oxide of aluminum. Probably because of it, sapphire does not change the immune status of the patient. If metals and poly-crystalline materials used for the bearing surfaces have different speed of wear of the micro-sites it leads to the coefficient increase of the pair friction and wear increase then this effect with sapphire is absent.

Tribological investigations of the friction pairs of materials were conducted by the scheme of rotational friction with the contact geometry of "ball on disk" type. The flat disk was manufactured from the investigated material and the ball from the material of counter body (Fig. 2).
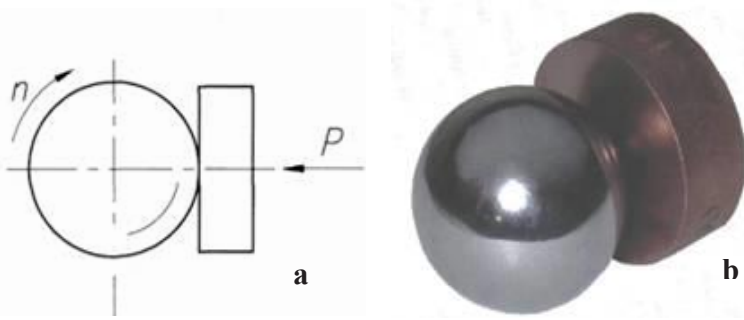

Fig. 2. Schematic arrangement of rotational friction $-\mathrm{a}$, and example of counter bodies $-\mathrm{b}$.

The obtained dependencies of the friction force of pairs: sapphire-sapphire, sapphire-ruby from the time of tests (number of cycles) has mainly non-monotonous abrupt character.(fig. 3-4) From them one can mark out three stages of wear, namely: running-in wear - creation of the work roughness and necessary supporting surface on frictional surfaces. At the moment of beginning of work the contact of bodies takes place in the point, accordingly the specific load is sufficiently high that leads to the abrupt growth of the friction force and because of wear of the surface material. 

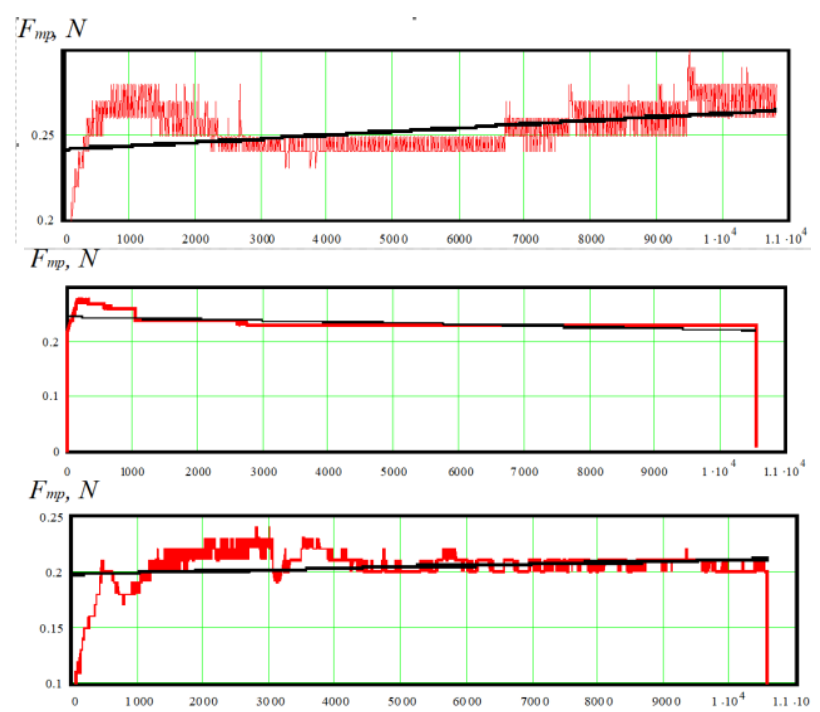

Fig. 3. Dependence of the friction force of the sapphiresapphire couple from the time of tests and its approximation by straight: flat $1018-\mathrm{a}$, flat $1080-\mathrm{b}$, flat $0001-\mathrm{c}$.

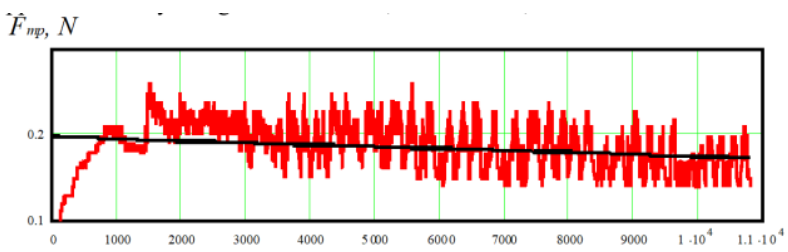

Fig. 4. Dependence of the friction force of the sapphire-ruby couple from the time of tests and its approximation by straight.

Normal wear is after achievement of the surface wear meaning, at which the optimal meanings of characteristics of supporting surface are reached, the stable process of friction of bodies has place with gradually decrease force of friction.

Catastrophic wear is in the process of friction of counter bodies of the wear products which fill the pockets on the friction surface and because of the weak (drop) feeding of the Ringer solution into the area of friction the wear products accumulate occupying all the vacant place in the pockets and as a result of this take part in the process of wear of the investigated surfaces as a free abrasive.

The pointed out above stages of wear can have different length of time or generally to be absent in the wear process.

From the dependencies follows that the best results in relation of the friction force to the pressing force shows the friction pair of sapphire-sapphire with orientation of the crystalline lattice on flatness 0001, linear wear of the friction pair of sapphire-sapphire with the orientation of the crystalline lattice on the flatness 0001 and sapphire-ruby.

Titanium and alloys on its basis are widely used in medicine as implants and other articles. From the point of view of biocompatibility for the implants, working for a long time in the live organism the use of titanium is preferable that unlike alloys does not contain alloying additives harmful for the live organism. However, titanium in its usual state has low mechanical properties in comparison with its alloys. This problem was solved by means of formation of Nano - and composition structure in technically pure titanium.

The use of the friction pairs of sapphire/titanium consisting of non-toxic materials would help us to solve the pointed out problem. However, for nowadays it is considered that from titanium and its alloys is impossible to manufacture a friction pair because of their high inclination to a contact snatch and therefore increased wear during friction. This property makes dangerous the use of titanium in the friction pairs.

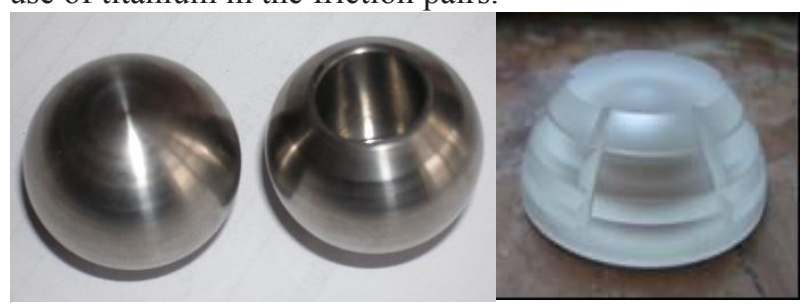

Fig. 5. Experimental specimens of titanium head and sapphire cup for endo-prosthesis of the hip-joint.

Thus, the work objective is the creation of a new bearing connection of endo-prosthesis of the hip-joint having the improved quality at the expense of use of hardened sapphire and biologically inert technically pure titanium with modification of the surface layer of IPD and subsequent nitrating as materials improving tribological properties of the connection.

a

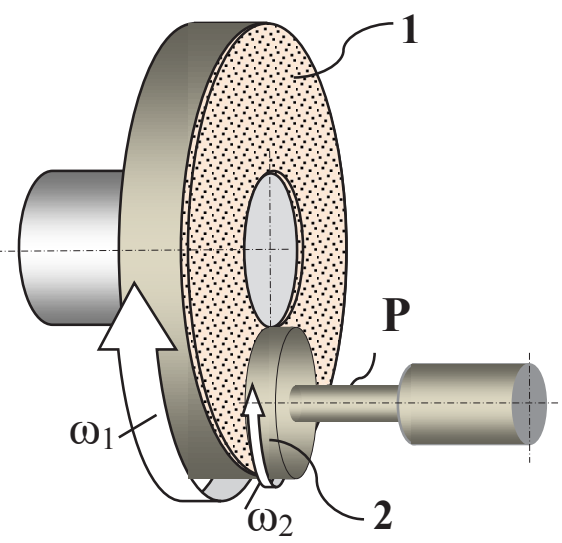

b

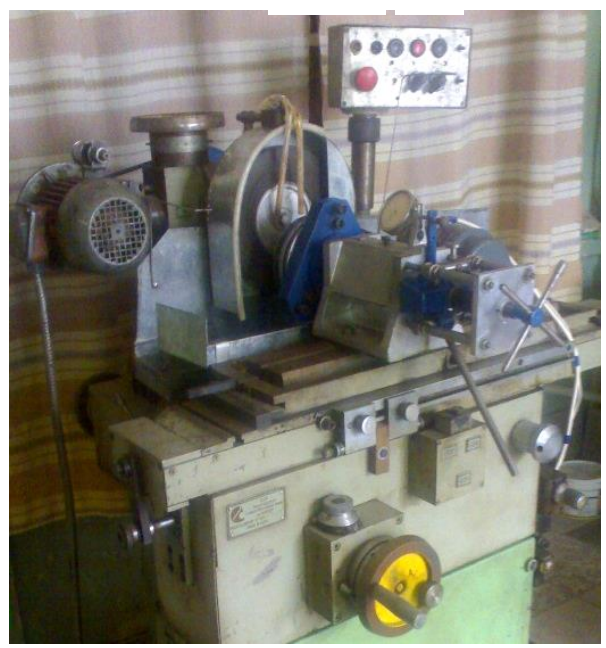

Fig. 6. a) Schematic arrangement of LPG: 1- Grinding ring, 2- Cassette with parts, b) Laboratory setting for LPG. 
For conduct of investigations of machinability of the sapphire crystal, the method of Low Temperature Precision Grinding (LPG) has been chosen developed at the Department of Mechanical Engineering of the Georgian Technical University as a version of progressive methods of diamond grinding of hard and brittle non-metallic materials.

In Fig.6a the machined parts set on the cassette in separators or by other method of mounting, for example, gluing carry out rotational movement with angular speed of $\omega_{2}$ and the grinding ring with speed of $\omega_{1}$ in the same direction. In the cutting area, the pressing is carried out by force $\mathrm{P}$ of the machined surfaces of parts to the work surface of the grinding ring.The experimental investigations were conducted on the laboratory setting equipped with the special precision head (Fig. 6b).

By the data analysis of complex, all-round experimental investigations conducted by us one can make the following conclusion:

By other equal conditions of machining, the most intractable is orientation 0001. For all the tested diamond rings, the ration of meanings of the linear removal of material $\mathrm{q}$ is within $\mathrm{q}_{0001} / \mathrm{q}_{1010}=0,25 \ldots 0,5$, at that, $\mathrm{q}_{1012} / \mathrm{q}_{1010}=0,75 \ldots 1$.

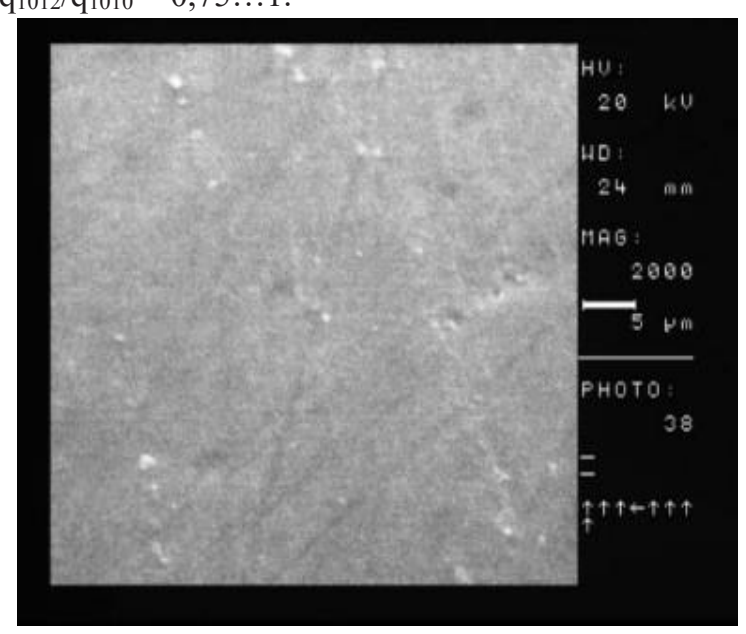

a

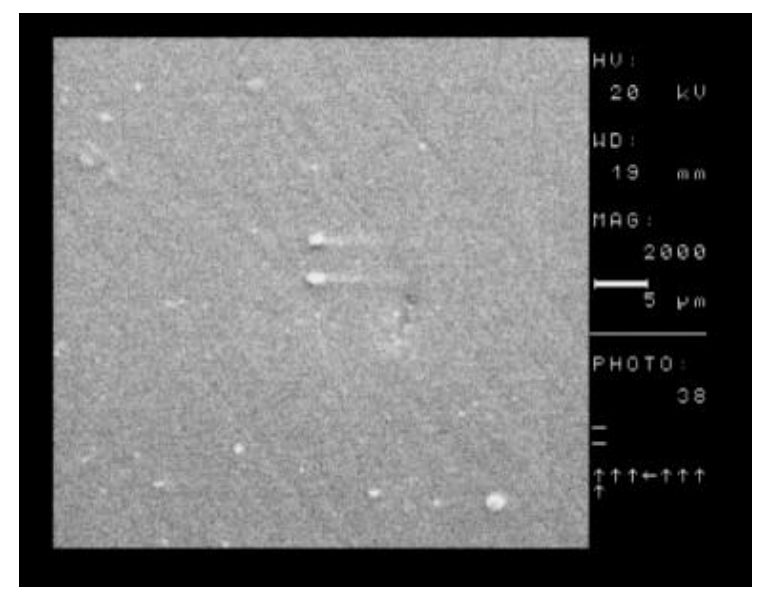

b

Fig. 7. Micro-photographs of surfaces of experimental specimens of sapphire machined by the LPG method: $\mathrm{a}$ - Orientation 1010, b - 1012. Diamond ring-ACM $14 / 10$, bunch organic special, $50 \%$. Cutting modes: $\mathrm{V}=1 \mathrm{~m} / \mathrm{sec}, \mathrm{P}=750 \mathrm{kPa}$.
The productivity of machining grows in the range of cutting speed of $\mathrm{V}=1 \ldots 6 \mathrm{~m} / \mathrm{sec}$ while at the further increase of the cutting speed to $12 \mathrm{~m} / \mathrm{sec}$ it remain constant.With increase of the pressing force $\mathrm{P}$ in all the investigated range the productivity of machining grows, however, in the interval of $\mathrm{P}=1000 \ldots 1500 \mathrm{kPa}$ the productivity growth considerably decreases. From characteristics of the diamond tool on productivity by the prevailing way influence granularity and bunch of the diamond tool. The concentration influence is insignificant. With the increase of the grain size within $\mathrm{d}_{3}=14 / 10 \ldots 28 / 20$ the productivity grows $1.5 \ldots 2.5$ times. The maximum productivity of machining is provided by the tool on ceramic bunch $(\{0001\}-130 \mathrm{mkm} / \mathrm{min}$; $\{1010\}-300 \mathrm{mkm} / \mathrm{min} ;\{1012\}-250 \mathrm{mkm} / \mathrm{min})$, then on metallic $(\{0001\}-50 \mathrm{mkm} / \mathrm{min} ;\{1010\}-200 \mathrm{mkm} / \mathrm{min}$; $\{1012\}-170 \mathrm{mkm} / \mathrm{min})$ and organic $(\{0001\}-$ $30 \mathrm{mkm} / \mathrm{min} ; \quad\{1010\}-120 \mathrm{mkm} / \mathrm{min} ; \quad\{1012\}-$ $110 \mathrm{mkm} / \mathrm{min}$ ). The tool on the ceramic bunch works in the mode of self-grinding [3, 4].

From characteristics of the diamond, tool the granularity and material of the tool bunch influence in prevailing way on the surface quality. With the grain increase in the investigated range the height of unevenness $\mathrm{R}_{\mathrm{z}}$ grows within $1 \ldots 1.5$ classes and the depth of the violated layer $\mathrm{H} 1.5 \ldots 2$ times. In other equal conditions of machining on orientation, (0001) higher quality of the surface is reached than on the rest two. The difference is in $1 \ldots 1.5$ classes of roughness. By this indication, the best results are shown by the diamond rings on organic bunch. So, for example, on the diamond rings on bunches BC-11 and organic special are obtained the following results: $\mathrm{R}_{\mathrm{z}}=0,25 \mathrm{mkm} ; \mathrm{t}_{\mathrm{P} 03}=35 \ldots 45 \% ; \mathrm{H}=2 \ldots .5$ $\mathrm{mkm}$. At that the meaning of parameter $\mathrm{Rz}$ is in order below than parameter $t_{\mathrm{P} 03}-1,5$ times higher of parameter $\mathrm{H}-3 \ldots 5$ fewer than the meanings of corresponding parameters that are obtained on the diamond rings on ceramic and metallic bunches.

The influence character of the process factors on output parameters for the chosen orientations of the sapphire crystal ((0001), (1010), (1012)) is constant.

By study of morphology of the machined surface the possibility of cutting the sapphire material by plastic deformation of the removed layer at low cutting speeds $\mathrm{V}=1 \ldots 3 \mathrm{~m} / \mathrm{sec}$ with the least depth of the violated subrelief layer has been proved (Fig. 7).

\section{Kinematics of forming the spherical heads of endo-prosthesis}

It is known that machining of vitreous materials, particularly, the sapphire crystal by means of plastic deformation of the removed layer instead of fragile destruction - dispersion, the pledge of acquisition of the machined surface practically without hereditary defect without sub-relief layer. Value H appeared to be the least namely on these specimens of sapphire. The obtained meaningful result requires individual investigations the conduct of which is being planned.

The development of a new or updating of the existing technological process of machining of the 
sapphire head sets the actual task of creation of new highly effective schemes of formation. The optimization criteria of technological operations such as productivity, indices of the surface quality and precision of machining determine the place of new schemes-methods of formation in technological process taking into account their advantages.

The schematic arrangement of the spherical head of endo-prosthesis is shown in Fig. 8. The partial sphere is determined by its radius and angle $\beta$ of spherical segment. There are several ways of machining of the spherical head. The closest to the LPG process in kinematics is the scheme of grinding of the partial sphere with use of the end grinding ring. The machined part rotates with angular speed of $\omega_{2}$ and $\omega_{3}$ around axes 2 and 3 subsequently (Fig. 9). This kinematics forming the incomplete surface of the sphere is taken as the basis of development and possible realization of more effective schemes of grinding of spherical heads of endo-prosthesis taking into account the kinematics and other positive features of the LPG method $[5,6]$.

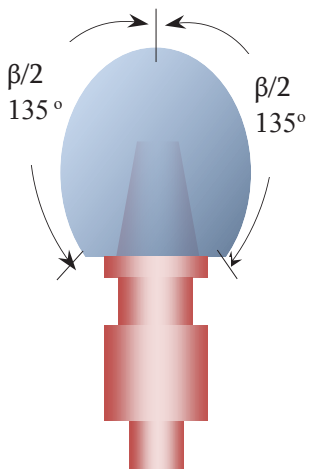

Fig. 8. Endo-prosthesis heads: $\beta$ - angle of spherical segment.

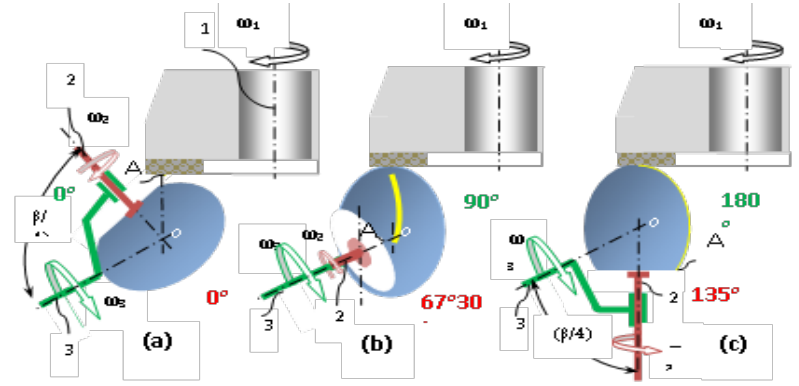

Fig. 9. Schematic arrangement of grinding of the partial sphere with use of the grinding ring.

The end grinding ring rotates with the angular speed of $\omega_{1}$ removing the tightness for machining from sphere with the required speed of cutting and rate of feeding. For full machining, the spherical head must rotate simultaneously around axes 2 and 3 with the angular speed of $\omega_{2}$ и $\omega_{3}$. Axes 2 and 3 intersect in the centre of sphere in the point $O$. As it is seen in Fig.4, the angle between axes, 3 and 4 must be equal to $\beta / 4$ but for to machine the whole surface of partial sphere angular speeds $\omega_{2}$ and $\omega_{3}$ must cinematically correlate as the both of them specify the values of components of the feeding rate. The linear speed on the ring/ball juncture and correlation of angular speeds $\omega_{2} / \omega_{3}$ will determine the surface texture of the half-complete or complete head of endo-prosthesis. The detailed analysis of kinematic connections between rotational speeds and other relative parameters of the grinding process are out of the Report and this will be discussed in the following publications. One of the formation schemes of the incomplete spherical surface with the forming grinding ring is shown in Fig. 10.

The partial spherical head rests on the forming grinding ring consisting of internal and external cones with abrasive layers (Fig. $10 \mathrm{a}, \mathrm{b}$ ) and rotates around axes 2 and 3 with angular speeds of $\omega_{2}$ and $\omega_{3}$ in the same manner as it was described above in Fig.8. The machined part by means of a strong spring $\mathrm{P}$ is pressed to the forming ring and is continuously fed to the required depth in direction of the ring. The applied pressing is equally distributed on the end of the ring work i.e. in points A and B (Fig. 8 b). In Fig. 8a are shown eight subsequent positions of the spherical head in one cycle. For clarification all, the positions are equally distributed along the ring although such subsequent positions are in the same place M. This scheme enables also carry out machining in "machining stations" around the ring. The main defect of this scheme is a complexity of the grinding ring and difference of speeds in points $\mathrm{A}$ and $\mathrm{B}$. The latter can be compensated in the same way as in the LPG process.

Comparatively simple schemes of formation of the spherical heads of endo-prosthesis with the use of commercially available shapes of the ring are shown below in Fig.9 a, b, c and d. The schemes presented in Fig.9 a, b and c differs only by the shape of used rings. The kinematics of formation of the partial spherical head is the same as the first scheme presented in Fig. 7 and 8.

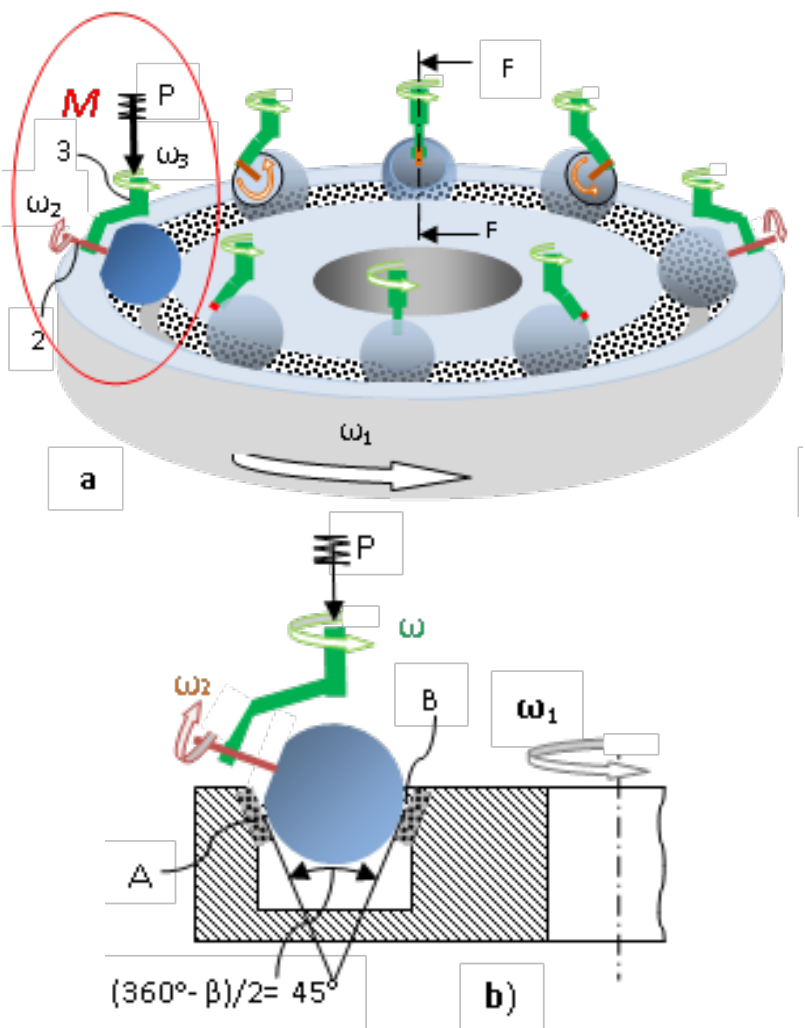

Fig. 10. Schematic arrangement of formation of the partial spherical head by the forming grinding ring (based on the LPG method). 


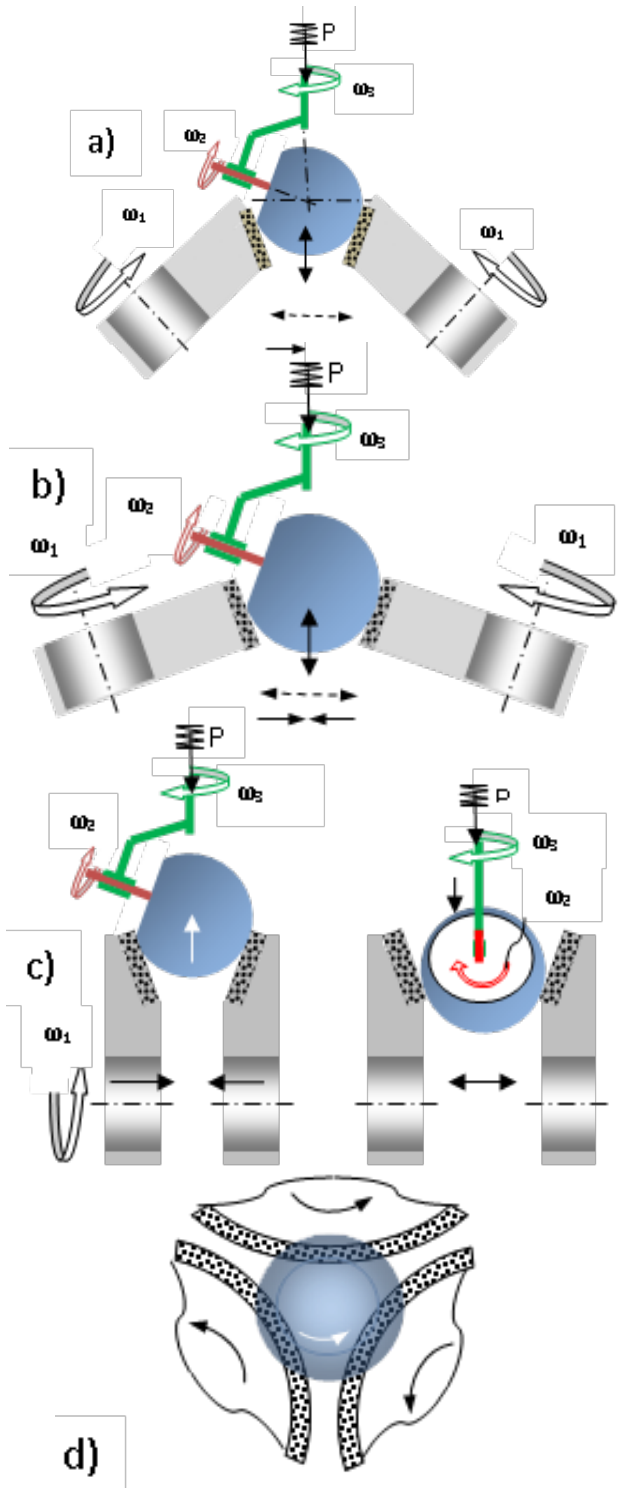

Fig. 11. Possible versions of grinding of the endo-prosthesis during the use of commercially available usual rings.

\section{Conclusion}

Scale of influence of single-crystal sapphire of the crystallographic flat orientation and the grinding conditions in the mode of removal of material, surface finishing and the state of sub-surface layer studied during low-temperature precise grinding have revealed that workability of single-crystal sapphire considerably depends on the crystallographic flat orientation. The relative values of removal of the material against the specimen with crystallographic flat orientation (1010) are within $\mathrm{q}_{0001} / \mathrm{q}_{1010}=0.25 \ldots 0.5$, and $\mathrm{q}_{1012} / \mathrm{q}_{1010}=0.75 \ldots 1$. In other equal conditions of the LPG process, the high quality of the ground surface is achieved for crystal orientation (1010). The difference with two other orientations of the crystallographic flatness is within 1 ...1.5 class of roughness.

By the studies of morphology of the ground surface was proved the grinding possibility by single-crystal sapphire in compliant mode, i.e. removal of the deformed layer by cutting without cracks at the low speed of cutting. In such conditions of machining was detected the least depth of the faulty sub-surface layer.

The structural schemes of formation of the partial spherical heads of endo-prosthesis for human hip-joint need a further analysis and optimization with the aim of designing of advanced technological processes and the prototype of grinding machines.

There are proposed two variants of the kinematic scheme of a special grinding machine for obtaining incomplete spherical surfaces of high accuracy, of which the first option is based on the use of a diamond grinding wheel with a complex shaped profile of the working part, and the second involves the use of grinding wheels with simple profiles, but the machine itself must have a software control system of high accuracy and reliability. The choice of the customer will depend on the required productivity of the machine and the level of qualification of the maintenance staff.

A great application of modern nanomaterials in this area is also expected [7].

\section{References}

1. B.I. Batiashvili, D.S. Butskhrikidze, G.A. Mamulashvili, R.S. Turmanidze, K. Kromp, B. Mills, O. Mgaloblishvili, Technological Possibilities of Low Temperature Precision Grinding Process when Machining Hard and Brittle Materials. Fractography of advanced ceramics, International Conference, Stará Lesná, High Tatras, May 2001.

2. R.S. Turmanidze, D.S. Butskhrikidze, K. Kromp, B. Mills, "Low temperature precision grinding of hard and brittle materials". Problems of Mechanics and Physico-Chemistry of the Process of Abrasive Machining, Kiev, pp. 490-499 (2002).

3. O.A. Rozenberg, S.V. Sokhan, V.V. Vozny, A.G. Mamalis, J. Gavlik, D.-J. Kim, Trends and Development in the Manufacturing of Hip Joints: An Overview. Int. J.Adv. Manuf. Technology, 27, pp. 537-542 (2006).

4. J.R. Lieberman, Two Alternative Bearings for Total Hip Arthroplasty: More Data Are Needed. J Am Acad Orthop Surg, pp. 61-62 (2009).

5. E. Dobrovinskaya, L. Litvinov, V. Pishchik, Monocrystals of corundum - K: Science, 256 pp. (1994).

6. R.S. Turmanidze, Implants of the human hip joints and peculiarities of their manufacture with the high precision and quality of machining of the work surfaces. Proceedings of the 13th International Conference on Tools (ICT-2012), 27-28 March 2012. University of Miskolc, Hungary. (2012).

7. P. Dašić, D.Lj. Mirjanić, Basic properties and applications of graphene. Proceedings of the Conference on Contemporary Materials (CM-2017), 9-10 November 2017. Banja Luka: Academy of Sciences and Arts of the Republic of Srpska, 2018, pp. 557-568. (In Serbian). 